\title{
Editorial Introduction to the Special Issue on Neuropsychological Interventions
}

\author{
Justin B. Miller ${ }^{1} \cdot$ Nicola J. Gates ${ }^{2}$
}

Received: 19 August 2016 / Accepted: 1 September 2016 /Published online: 23 September 2016

(C) Springer Science+Business Media New York 2016

As the numbers of adults affected by neurocognitive disorders rise, there is an increasing requirement for neuropsychological interventions to maximize quality of life for patient and family, and to decrease the economic burden associated with specialized long-term care (Huckans et al. 2013). Consequently, there is a critical need for evidence-based techniques that combine the best available research evidence with clinician expertise in providing care to individual patients (Chelune 2010).

While neuropsychologists traditionally undergo robust training in cognitive and behavioural assessment and focus their professional efforts on evaluation and diagnosis, many neuropsychologists are increasingly integrating this core training with intervention techniques to compensate for, or mitigate the effects of, neurologic disease. Provision of interventions involves both functional and foundational core competencies according to recent models (Stucky et al. 2010). However, training and practice in neuropsychological interventions has been widely neglected, for example, in the United States where less than $48 \%$ of neuropsychologist respondents reported receiving specific training in intervention skills (Shultz et al. 2014).

This special issue of Neuropsychology Review highlights diverse interventions for two important clinical populations with neurocognitive disorders, namely, people with geriatric and psychiatric conditions. The purpose of this special issue of

Justin B. Miller

justin.b.miller@gmail.com

1 Cleveland Clinic Lou Ruvo Center for Brain Health, Las Vegas, NV, USA

2 Centre for Healthy Brain Aging, Faculty of Medicine, University of New South Wales, Randwick, NSW, Australia systematic reviews is to increase scientific knowledge regarding neuropsychological interventions for both clinicians and researchers.

The first two papers target early cognitive change associated with progressive dementia. Mild cognitive impairment (MCI), which is often prodromal Alzheimer disease, has been a focus of numerous clinical trials with particular emphasis on cognitive outcomes. Unfortunately, many trials have paid considerably less attention to non-cognitive factors that could demonstrate generalization of treatment benefits, although non-cognitive factors are often included as secondary endpoints. Chandler and colleagues review non-pharmacological intervention studies in older adults with MCI, specifically studies that report what Chandler and colleagues describe as "generalizability outcomes," including activities of daily living (ADLs), mood, quality of life (QOL), and metacognitive outcomes. A central finding of Chandler and colleagues review is that, while there may be some promise among the interventions studied, heterogeneity among both interventions and outcomes hinders efforts to synthesize findings across studies into a meaningful pattern of conclusions.

Mowszowski and colleagues also set out to study cognitive changes following intervention in older adults. These authors were interested in both adults with MCI and cognitively healthy subjects, targeting executive functioning using a strategy-based cognitive training paradigm. Although their inclusion and exclusion criteria led the authors to retain so few studies of adults with MCI that systematic review or meta-analysis were not feasible, Mowszowski and colleagues' review of the healthy adult literature revealed wide-ranging but mostly positive - treatment effects. Similar to Chandler and colleagues, methodological heterogeneity among the primary studies remains an important limitation of the current literature, precluding clear inferences of benefit.

In a different patient population, Sandroff and colleagues reviewed exercise and physical activity effects on cognition in 
people with multiple sclerosis. Sandroff and colleagues found tentative evidence to suggest a positive effect of physical activity. Interestingly, although the overall finding of their systematic review is that the literature does not provide unambiguous results, the uncertainty of effects appears to be partially related to the design of the primary studies reviewed. When the scope of the review is limited to studies utilizing cognition as a primary outcome as opposed to a secondary outcome, a more consistent pattern of support is apparent. While the quality of evidence in the reviewed studies tends to be low, the consistency of results across studies is noteworthy and highlights the need for more rigorous studies.

The final two papers in this special issue review interventions for neuropsychiatric syndromes. Martin and colleagues report cognitive outcomes following treatment with transcranial magnetic stimulation (rTMS) in adults across a diverse range of neuropsychiatric conditions. With the exception of working memory, where a small positive treatment effect was noted in patients with schizophrenia, rTMS failed to improve cognition in any of the other populations studied. While each study targeted the dorsolateral prefrontal cortex (DLPFC) for stimulation, the treatment parameters and targeted outcomes were quite variable across studies. Thus, a common theme across reviews is the lack of consistent effects from which to draw clear conclusions regarding treatment efficacy.

Finally, Campos and colleagues reviewed the neuroplastic effects of social cognitive training among individuals with schizophrenia spectrum disorders. Not only did they observe robust effects following social cognitive training, behavioral changes were evident as well, supporting the utility of intervention in this population. What distinguishes this review is the narrow scope of outcome measures. Although none of the studies reviewed utilized the same experimental task, interpretation of the neuroplasticity effects across studies was complicated by the use of multiple structural and functional outcomes.

There are several notable themes that emerge from this series of reviews. The diversity of interventions carried out is remarkable and reflects the flexibility and richness of neuropsychologist expertise in understanding brainbehaviour relationships. Beyond the variable patient populations, the types of interventions ranged broadly from skills training, to cognitive training, to improvement of daily living skills.
It is equally clear that greater uniformity in study methods is necessary to facilitate meaningful comparison, interpretation and aggregation of results from studies seeking to demonstrate treatment effects in neuropsychology. It is perhaps no coincidence that the single review describing consistent evidence of a treatment effect is the one review in which the diversity in outcome measures is limited. This observation does not suggest that researchers should always adopt the same experimental methods, but rather, underscores the importance of integrating common data elements with known sensitivities whenever practicable to facilitate subsequent interpretation of outcomes, whether subject to systematic review or not. It is also important that study authors adhere to established guidelines to maximize transparency and reporting accuracy, a mechanism which will further facilitate interpretation and aggregation of findings across primary studies. There are several well-established guidelines available that are specific to primary study type (e.g., CONSORT for clinical trials, STROBE for observational studies: see www.equatornetwork.org). Additionally, we suggest that intervention methods are clearly described including specific details of 'dose' of intervention (time per session, frequency per week, intensity where relevant, and duration of treatment) to facilitate review and replication with the ultimate goal of clinical implementation of techniques where efficacy has been demonstrated.

\section{References}

Chelune, G. J. (2010). Evidence-based research and practice in clinical neuropsychology. The Clinical Neuropsychologist, 24(3), 454-467. doi:10.1080/13854040802360574.

Huckans, M., Hutson, L., Twamley, E., Jak, A., Kaye, J., \& Storzbach, D. (2013). Efficacy of cognitive rehabilitation therapies for mild cognitive impairment (MCI) in older adults: working toward a theoretical model and evidence-based interventions. Neuropsychology Review, 23(1), 63-80. doi:10.1007/s11065-013-9230-9.

Shultz, L. A., Pedersen, H. A., Roper, B. L., \& Rey-Casserly, C. (2014). Supervision in neuropsychological assessment: a survey of training, practices, and perspectives of supervisors. The Clinical Neuropsychologist, 28(6), 907-925. doi:10.1080 /13854046.2014.942373.

Stucky, K. J., Bush, S., \& Donders, J. (2010). Providing effective supervision in clinical neuropsychology. The Clinical Neuropsychologist, 24(5), 737-758. doi:10.1080/13854046.2010.49078. 